\title{
Enhancement of the Liquefaction Rate in Small-Scale Helium Liquefiers Working Near and Above the Critical Point
}

\author{
C. Rillo, ${ }^{1,2}$ M. Gabal, ${ }^{2,3}$ M. P. Lozano, ${ }^{2,3}$ J. Sesé, ${ }^{2,3}$ S. Spagna, ${ }^{4}$ J. Diederichs, ${ }^{4}$ R. Sager, ${ }^{4}$ C. Chialvo, ${ }^{4}$ J. Terry, ${ }^{4}$ \\ G. Rayner, ${ }^{4}$ R. Warburton, ${ }^{5}$ and R. Reineman ${ }^{5}$ \\ ${ }^{1}$ Instituto de Ciencia de Materiales de Aragón (ICMA), CSIC-Universidad de Zaragoza, \\ E-50009 Zaragoza, Spain \\ ${ }^{2}$ Departamento de Física de la Materia Condensada, Universidad de Zaragoza, E-50009 Zaragoza, Spain \\ ${ }^{3}$ Instituto de Nanociencia de Aragón (INA), Laboratorio de Microscopías Avanzadas (LMA), \\ Universidad de Zaragoza, E-50018 Zaragoza, Spain \\ ${ }^{4}$ Quantum Design, Inc., San Diego, California 92121, USA \\ ${ }^{5}$ GWR Instruments, Inc., San Diego, California 92121, USA
}

(Received 27 January 2015; revised manuscript received 31 March 2015; published 8 May 2015)

\begin{abstract}
Low-temperature research laboratories with typical liquid-helium consumption of the order of tens of liters per day have greatly benefited from the recent development of small-scale liquefiers. In general, these liquefiers are based on Gifford-McMahon or pulse-tube closed-cycle refrigerators with a nominal cooling power ranging from 1 to $1.5 \mathrm{~W}$ at $4.2 \mathrm{~K}$. The liquefaction rate for these cryocooler-based liquefiers depends on the pressure at which the helium is liquefied, although the final user conditions of the produced liquid helium are always atmospheric pressure and boiling temperature (e.g., $4.2 \mathrm{~K}$ at $100 \mathrm{kPa}$ ). Here, we show a systematic study on this effect, in which an enhancement in excess of $70 \%$ in liquefaction rate is found experimentally for pressures near and above the critical point of helium (220 kPa). We propose that the underlying mechanism for the liquefaction enhancement is based on the increase in cryocooler cooling power with temperature and the decrease of the helium enthalpy with pressure.
\end{abstract}

DOI: 10.1103/PhysRevApplied.3.051001

In recent years, there have been several dramatic liquidhelium shortages worldwide. These shortages, coupled with ever-increasing prices for a liter of liquid helium, have created operational challenges for a wide spectrum of scientific disciplines and technological areas. Liquid helium is, for example, necessary to operate superconducting magnets in medical research centers and hospitals, and is needed in many low-temperature physics, chemistry, and biology research laboratories, where helium recovery and liquefaction infrastructures do not exist [1]. The well-known commercial and industrial liquefaction plants derived from Collins technology [2] are available on the market and have been for many decades, but they are neither economically justified nor efficient when liquidhelium consumption is below $100 \mathrm{l} /$ day. Therefore, there is an increasing need for efficient, small-scale helium liquefiers and helium associated recovery technology [3].

During the last decade, several closed-cycle refrigeratorbased small-scale helium liquefiers [4] have been proposed and successfully commercialized as viable alternatives to the Collins liquefiers. In spite of their

Published by the American Physical Society under the terms of the Creative Commons Attribution 3.0 License. Further distribution of this work must maintain attribution to the author(s) and the published article's title, journal citation, and DOI. apparent simplicity, however, these liquefiers are not energy efficient, they suffer from helium losses, and are difficult to operate and transport to and from cryostats requiring helium refills.

In an attempt to address these shortcomings, we develop a versatile, small-scale liquefier [5-7] based on technology that achieves better liquefaction rates with lower energy consumption. This paper shows how better liquefaction rates are achieved and describes some fundamental thermodynamics aspects that are behind the advantage of working near and above the critical point.

These experiments are performed on a helium liquefier, model ATL160 from Quantum Design, that is schematically shown in Fig. 1. It incorporates a commercial twostage Gifford-McMahon (GM) cryocooler [8] that employs a 7-kW compressor, and has a nominal cooling power of $1.5 \mathrm{~W}$ at $4.2 \mathrm{~K}$ on the second stage [9]. The liquefier is comprised of a 160-1 Dewar with the cold head installed in its neck, a gas-handling controller, and ancillary components (pressure gauge, temperature sensor, level meter, and transfer port) to control all the stages of liquid production and liquid-handling processes.

One important feature of the advanced technology liquefiers (ATL) is the precise control of the pressure of helium gas inside the liquefier's Dewar by means of a forward pressure controller integrated with a mass flow meter. 


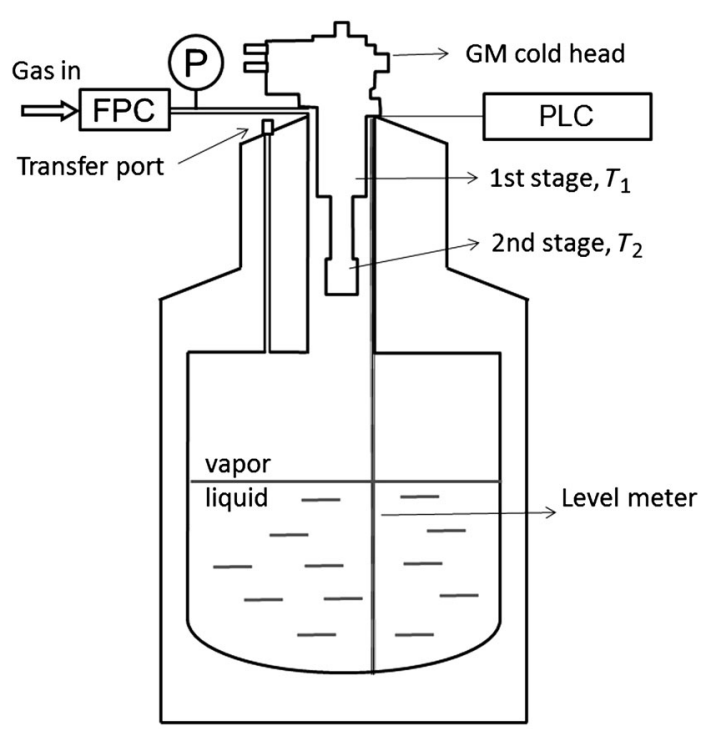

FIG. 1. Schematic representation of an ATL160 liquefier. The pressure $P$ of the helium inside the liquefier is controlled by means of a forward pressure controller (FPC). A programmable logic controller (PLC) reads $T_{1}$ and $T_{2}$ and the liquid-level meter probe. The PLC sets the desired liquefaction pressure and continuously reads actual $P$ and helium mass-flow values from the FPC.

For atmospheric pressure corresponding to sea level, $100 \mathrm{kPa}$, the enthalpy difference between gaseous helium at $300 \mathrm{~K}$ and liquid helium at $4.2 \mathrm{~K}$ is $1564 \mathrm{~kJ} / \mathrm{kg}$ [10]. Under these conditions, the production of 11 of liquid helium (density $=125 \mathrm{~g} / \mathrm{l}$ ) per hour requires a cooling power of $54 \mathrm{~W}$. In practice, however, this power is delivered not at a single point but rather is distributed along the length of the cold head in a temperature range between $300 \mathrm{~K}$ and $4.2 \mathrm{~K}$. Helium gas enters the liquefier at room temperature and it is first precooled by heat exchange with the first stage of the cold head to a temperature $T_{1}$ typically between 40 and $60 \mathrm{~K}$. Afterwards, the helium gas continues on its way down and is further cooled by heat exchange with the second stage, until the condensation temperature is reached when it converts into liquid. Experiments performed at different constant pressures, above and below atmospheric pressure, confirm that the second-stage temperature $T_{2}$ becomes constant during liquid production and it coincides with the temperature of liquid-vapor equilibrium on the saturation line of the equilibrium phase diagram, both for increasing and for decreasing pressures. Thus, the higher the liquefaction pressure, the higher the second-stage temperature, as presented in Fig. 2.

The performance of a two-stage cryocooler is typically represented by a cooling load map that represents the temperatures measured at both stages in vacuum when different heating powers are applied to the cryocooler's stages. The cold-head second stage can typically reach a minimum temperature of $2.5 \mathrm{~K}$ when no heating power is applied, but consequently the cryocooler has zero coolingpower capability at this base temperature. The available

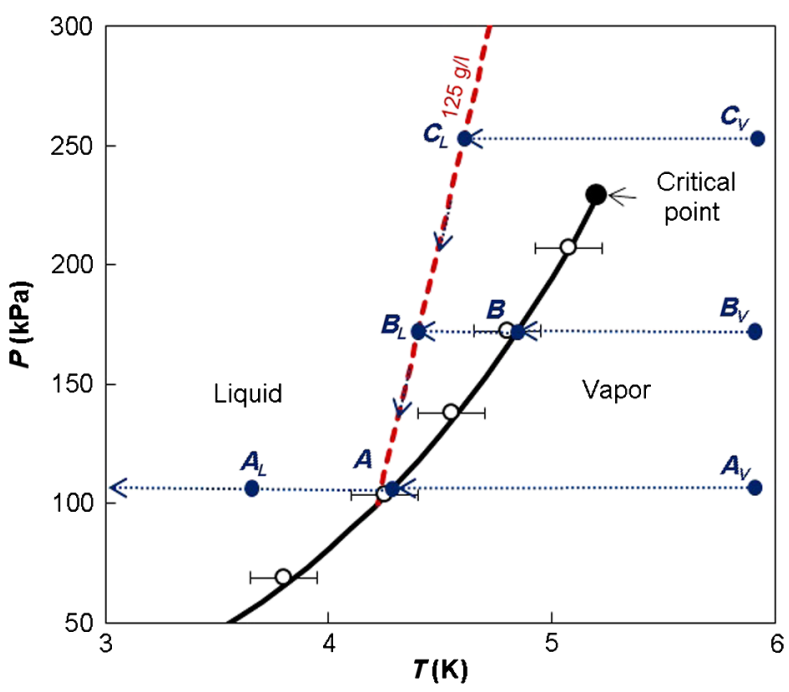

FIG. 2. Open circles are experimental points of the values of $P$ and $T_{2}$ measured when liquid is being produced in the liquefier. Solid line is the liquid-vapor coexistence line of the phase diagram of helium. Dashed red line corresponds to liquid helium with a constant density of $125 \mathrm{~g} / 1 . A_{V}, B_{V}, C_{V}$ represent vapor points. $A_{L}, B_{L}, C_{L}$ represent single-phase liquid points. Trajectories $A_{V} \rightarrow A \rightarrow A_{L}, \quad B_{V} \rightarrow B \rightarrow B_{L} \rightarrow A \rightarrow A_{L}$, and $C_{V} \rightarrow C_{L} \rightarrow B_{L} \rightarrow A \rightarrow A_{L}$, correspond to liquefaction at a slightly positive gauge pressure, $107 \mathrm{kPa}$, at an intermediate pressure, $170 \mathrm{kPa}$, and at a pressure above the critical pressure, $251 \mathrm{kPa}$, respectively. Liquefaction points $A$ and $B$, and the critical point, are indicated by closed circles on the two-phase liquid-vapor saturation line.

cooling power increases rapidly with temperature as shown in Fig. 3(a). For example, a cooling capacity of $1.5 \mathrm{~W}$ is available at $4.2 \mathrm{~K}$, whereas $4 \mathrm{~W}$ are available at $6 \mathrm{~K}$. An increase of cooling power with temperature is also observed in the first stage. The physical reason behind this effect is the thermal dependence of heat capacitance of the regenerator material that is at work in the displacers of the cold head [11].

The cold head inside the liquefier is not in vacuum, but in direct contact with the helium gas stream. Helium can therefore exchange heat not only with cold flanges of the first and second stages, where expansion volumes of the cold head are located, but also with the rest of the length of the cold head. The extraction of cooling power along the length of the cold head was previously observed and considered as extra cooling power added to the power existing in vacuum conditions [12-14].

Even though the cold head is not in vacuum, we can expect that a higher cooling power and, hence, a higher liquefaction rate should be reached if helium is liquefied at higher temperatures. This explains the results previously obtained using a sophisticated heat-exchanger design [15]. A further argument in favor of an expected higher liquefaction rate is that helium loses its enthalpy faster when it is being cooled down at a higher pressure. Figure 4 shows the enthalpy of helium in the region close to the condensation 

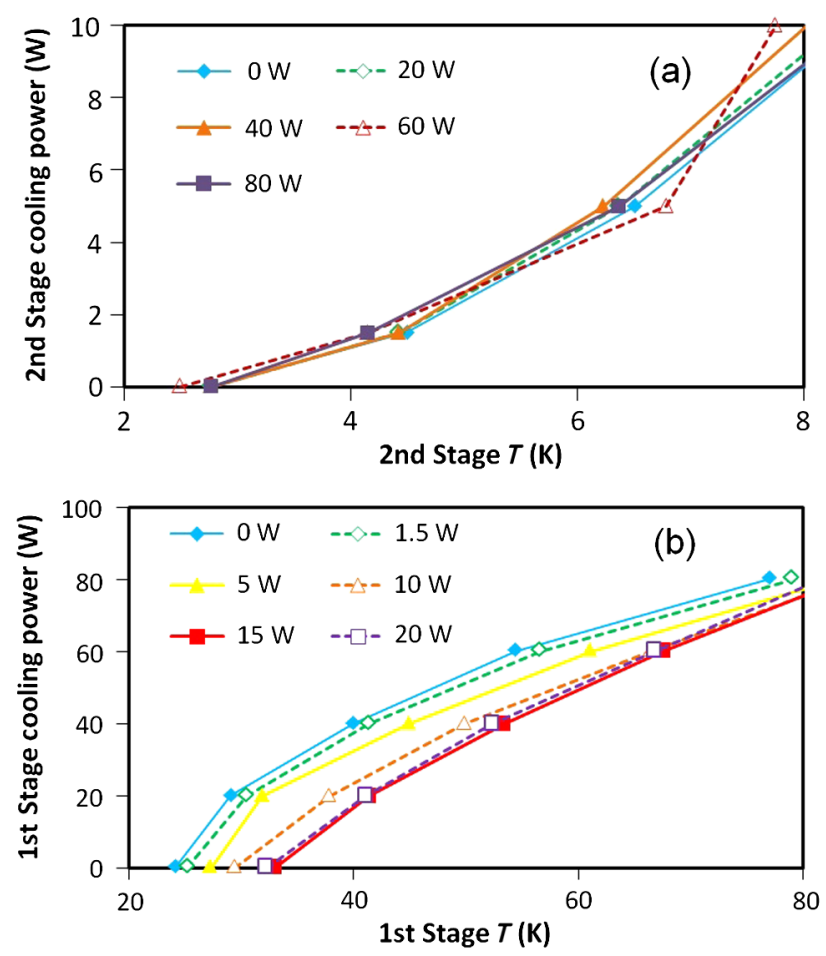

FIG. 3. (a) Cooling power in the second stage versus its temperature for different heating powers applied on the first stage. (b) Cooling power in the first stage versus its temperature for different heating powers applied on the second stage.

temperature for pressures $107 \mathrm{kPa}, 170 \mathrm{kPa}$, and $251 \mathrm{kPa}$. For any temperature, it shows that the enthalpy is lower when the pressure is higher and, hence, less heat has to be extracted by the cold head to reach the final desired point $A$ of $4.2 \mathrm{~K}$ and $107 \mathrm{kPa}$.

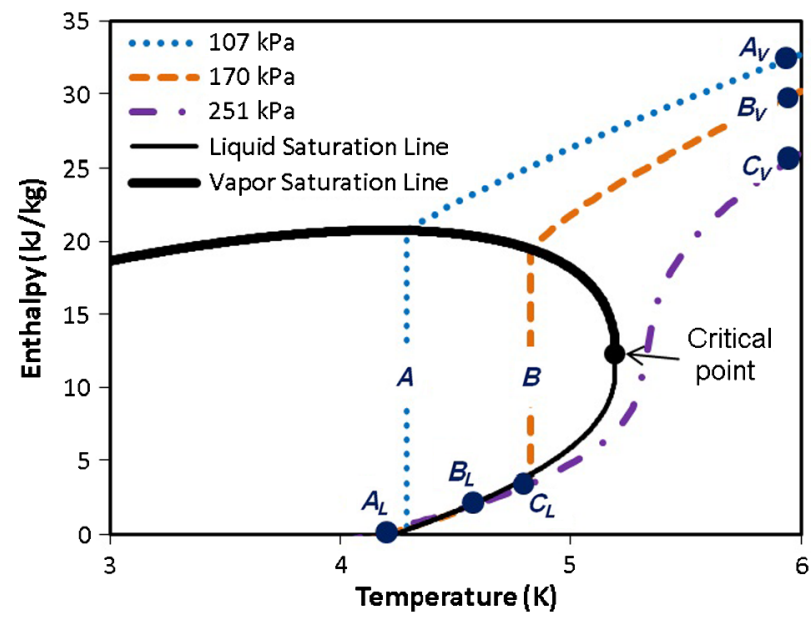

FIG. 4. Helium enthalpy corresponding to several absolute pressure values. The reference of zero enthalpy has been arbitrarily chosen to be at $4.2 \mathrm{~K}$ and $100 \mathrm{kPa}$ [10]. Trajectories $A_{V} \rightarrow A \rightarrow A_{L}, \quad B_{V} \rightarrow B \rightarrow B_{L} \rightarrow A \rightarrow A_{L}$, and $C_{V} \rightarrow C_{L} \rightarrow$ $B_{L} \rightarrow A \rightarrow A_{L}$ correspond to liquefaction at $107 \mathrm{kPa}, 170 \mathrm{kPa}$, and $251 \mathrm{kPa}$, respectively.
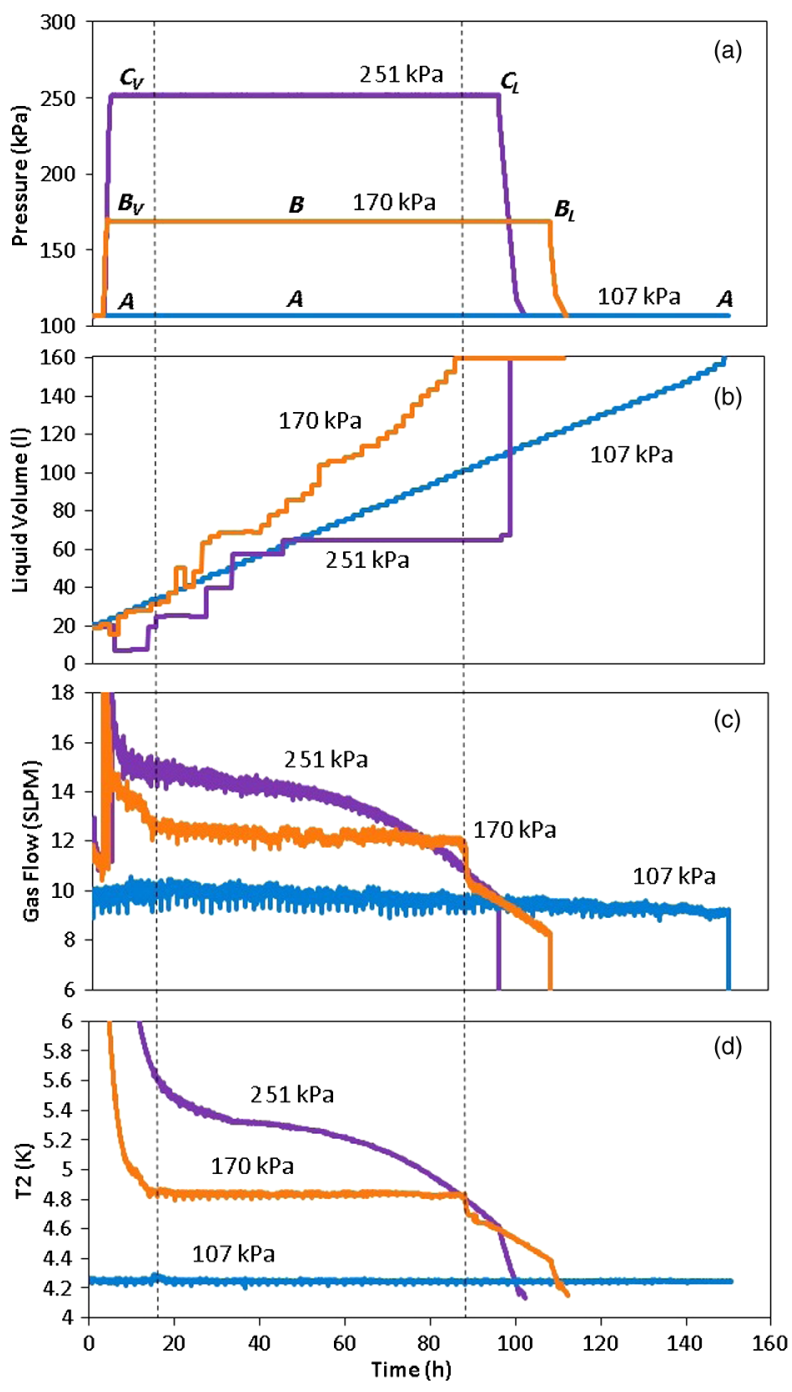

FIG. 5. Comparison of the time evolution of (a) pressure, (b) the liquid volume, (c) gas flow (standard liters per minute), and (d) $T_{2}$ for a liquefaction run performed at $251 \mathrm{kPa}$ (violet line), $170 \mathrm{kPa}$ (orange line), and $107 \mathrm{kPa}$ (blue line). Note that the starting condition in all cases is a partially filled Dewar with about 201 at $4.2 \mathrm{~K}$. Points $A, B, B_{V}, B_{L}, C_{V}$, and $C_{L}$ are indicated in (a). The vertical dashed lines indicate the liquefaction time interval at point $B(170 \mathrm{kPa})$.

Figure 5 compares experimental data recorded for three liquefaction runs at $107 \mathrm{kPa}, 170 \mathrm{kPa}$, and $251 \mathrm{kPa}$. The Dewar is partially filled in all cases with about 201 of helium at $4.2 \mathrm{~K}$. In the liquefaction experiment at $107 \mathrm{kPa}$, the helium gas is cooled down along the line $A_{V} \rightarrow A$, and liquid with a density of $125 \mathrm{~g} / 1$ is produced at point $A$ of the phase diagram of Fig. 2, corresponding to a temperature of $4.2 \mathrm{~K}$, as experimentally shown in Fig. 5(d). On the other hand, the production of liquid for the liquefaction at $170 \mathrm{kPa}$ occurs at point $B$ of Fig. 2, at a temperature of $4.8 \mathrm{~K}$. Figure 5(b) shows how the Dewar is filled with liquid helium at a much faster rate when the pressure is $170 \mathrm{kPa}$. Note that some erratic behavior in the liquid level meter occurs at high pressures. The reason for this 
behavior has to do with the fact that superconductinglevel-meter readings are based on the difference between liquid and gas, and the properties of liquid and gas become more similar when the critical temperature is approached. Under these conditions, the Dewar is already full of liquid at the 85-h mark while at a liquid temperature of $4.8 \mathrm{~K}$. From there on, the liquefaction run continues further to cool down the liquid to reach the point $B_{L}(170 \mathrm{kPa}, 4.4 \mathrm{~K})$ in Fig. 2 ; at point $B_{L}$, the liquid density equals that of point $A$. Figure 5(c) shows that, during this process, a considerable helium gas flow is entering the liquefier, accounting for the difference in density between liquid at $4.8 \mathrm{~K}$ and $4.2 \mathrm{~K}(115 \mathrm{~g} / 1$ and $125 \mathrm{~g} / 1$, respectively). When compared with data taken at $107 \mathrm{kPa}$ and a liquid temperature of $4.2 \mathrm{~K}$, we can see how the liquefier operating at high pressure takes about 2 days less to be filled with liquid helium.

A third liquefaction run is performed at $251 \mathrm{kPa}$, above the critical point. In this case, gas is cooled through the supercritical state, from point $C_{V}$ continuously down to point $C_{L}(251 \mathrm{kPa}, 4.6 \mathrm{~K})$, well inside the liquid region (see Fig. 2), without undergoing a phase transition. At that temperature, flow is interrupted so that pressure and temperature follow the trajectory $C_{L} \rightarrow A$ with constant density.

The above experimental results and the corresponding liquefaction rate enhancement can be better understood with typical gas-flow-versus-time diagrams (Fig. 6) for three complete liquefaction runs. Two of these runs are at the pressures and temperatures corresponding to points $A$ and $B$ of the helium phase diagram, on the saturation line (Fig. 2), with trajectories $A_{V} \rightarrow A$ and $B_{V} \rightarrow B \rightarrow$ $B_{L} \rightarrow A$ respectively. The third run is above the critical point (i.e., $P>P_{C}$ ), corresponding to the trajectory $C_{V} \rightarrow C_{L} \rightarrow B_{L} \rightarrow A$.

In general, for a pressure below the critical pressure $\left(P_{C}\right)$ and for specific starting conditions (starting from room temperature, or with the Dewar already cold but empty of liquid, or with a given amount of liquid at atmospheric

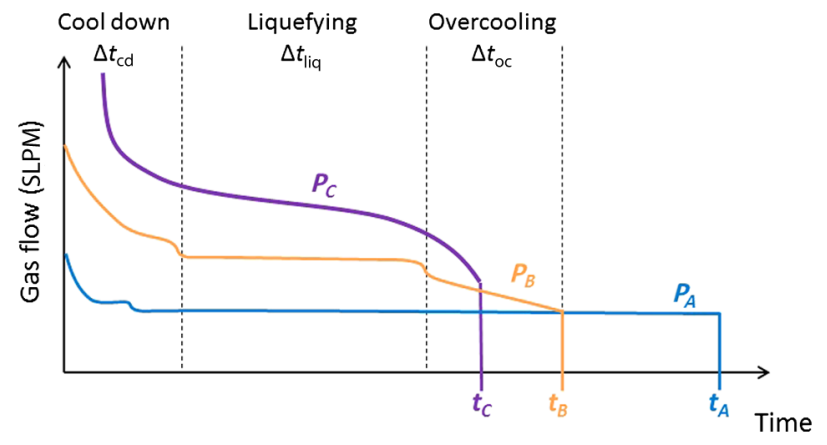

FIG. 6. Representation of the typical helium flow for liquefaction runs at pressures $P_{A}, P_{B}$, and $P>P_{C}$ corresponding to liquefaction points $A, B$, and line $C_{V} \rightarrow C_{L}$ in the phase diagram of Fig. 2. The time periods corresponding for vapor cooldown, liquefying, and liquid overcooling are indicated for the case of $P_{B}$. pressure), a liquefaction run can be divided in three distinct time periods. (1) Pressure is built up and vapor cool-down time period $\Delta t_{\text {cd }}$ occurs, during which the vapor increases its density from that of its initial temperature and pressure at points somewhere in the vapor region $A_{V}$ or $B_{V}$ till the corresponding saturation values at the respective points $A$ and $B$ in Fig. 2. In fact, this process corresponds to the isobaric cooling trajectories represented in Fig. 2, from the vapor region till the temperature reaches the saturation line, i.e., $A_{V} \rightarrow A, B_{V} \rightarrow B$, respectively. (2) Liquefying time period $\Delta t_{\text {liq }}$ occurs, during which saturated liquid is produced at constant pressure and temperature at points $A$ or $B$. And, (3) once the Dewar is full of saturated liquid, overcooling takes place during a time period $\Delta t_{\mathrm{oc}}$ that corresponds to the isobaric cooling trajectories $A \rightarrow A_{L}$ (with $\left.A=A_{L}\right), B \rightarrow B_{L}$ in Fig. 2. The final step is the pressure and temperature reduction $B_{L} \rightarrow A$ at constant density.

As already explained, for a liquefaction pressure above the critical pressure, the liquid is produced without undergoing a phase transition through the saturation line but by a continuous vapor-densification process through the supercritical region. For all cases in which $P>P_{A}$, the final cooldown and corresponding simultaneous pressurereduction process (at about $25 \mathrm{kPa} / \mathrm{h}$ in the above described experiments) takes place after the density of the fluid reaches the $125 \mathrm{~g} / 1$ line (e.g., at points $C_{L}$ and $B_{L}$ ). The total liquefaction run time is represented by $t_{A}, t_{B}$, or $t_{C}$ in Fig. 6 for liquefaction at points $A$ and $B$ or for liquefaction through the supercritical line $C_{V} \rightarrow C_{L}$, respectively. It is noteworthy that $t_{A} \gg t_{B}>t_{C}$. The area between any of two gas-flowversus-time curves, corresponding to two liquefaction runs on different saturation points of the phase diagram, is directly related to enhancement of the liquefaction rate.

Notably, if gas flow is not interrupted at A, high-density, nonboiling, liquid helium is obtained at atmospheric pressure and temperatures down to the base temperature of the cold head $(4.2 \mathrm{~K}>T>2.5 \mathrm{~K})$, i.e., any point $A_{L}$ to the left of point $A$ in Fig. 2. The production of single-phase liquid helium (trajectory $A \rightarrow A_{L}$ ) allows liquid transfers with losses as low as $3 \%$, providing a clear advantage with respect to the typical 20-25\% transfer losses obtained when transferring saturated two-phase (boiling) liquid helium at $4.2 \mathrm{~K}$.

The experimentally measured enhancement of liquefaction rate with the liquefaction pressure is finally seen in Fig. 7. There, we represent the complete duration of many liquefaction runs (several hundreds) performed at different liquefaction pressures, but always starting with the empty Dewar at $4.2 \mathrm{~K}$ and finishing with a full Dewar at $4.2 \mathrm{~K}$ and $100 \mathrm{kPa}$.

An important decrease in the total duration of the liquefaction runs is observed when the liquefaction pressure is increased. The decrease corresponds to more than $70 \%$ increase in the average liquefaction rate, from about $201 /$ day to above 351 /day at the highest tested pressures. 


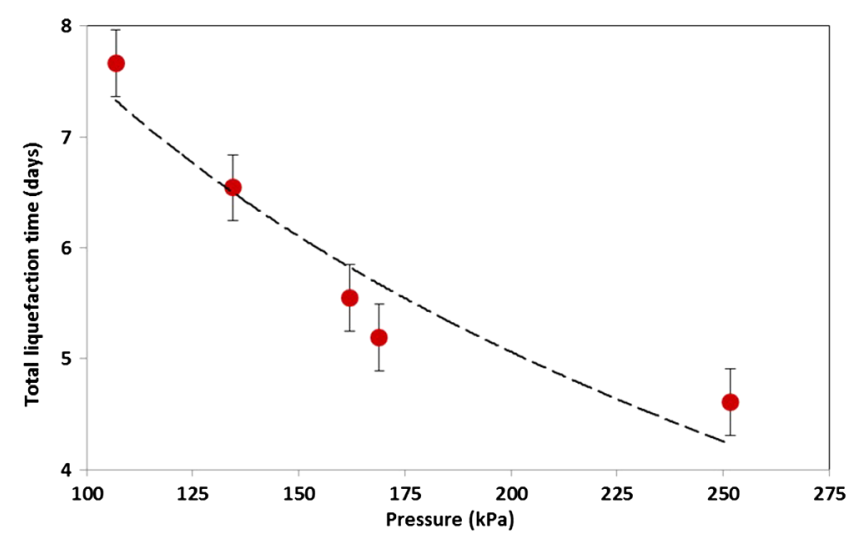

FIG. 7. Total duration of a complete liquefaction run, as a function of the liquefaction pressure. The starting point is an empty Dewar at $4.2 \mathrm{~K}$ and $100 \mathrm{kPa}$ and the run finished with a full Dewar with 1601 of helium at $4.2 \mathrm{~K}$ and $100 \mathrm{kPa}$. The error bars indicate the dispersion of the analyzed data from hundreds of liquefaction runs. The dashed line is a guide for the eye. Helium with purity $>99.999 \%$ is used.

The reduction in the total duration of a liquefaction run is proportional to the liquid volume produced. Therefore, it is always advantageous to extract as much liquid as possible before starting a new high-pressure liquefaction run.

To conclude, this paper describes a versatile helium liquefier that offers improved efficiency versus traditional small-scale liquefier offerings through high-pressure liquefaction.

The authors would like to acknowledge the use of Servicio General de Apoyo a la Investigación-SAI, Universidad de Zaragoza. Financial support from the Spanish Ministry of Economy and Competitiveness through Project No. IPT2012-0442-420000, in addition to European Union FEDER funds, is gratefully acknowledged.

[1] W. P. Halperin, The impact of helium shortages on basic research, Nat. Phys. 10, 467 (2014).

[2] S. C. Collins, Helium refrigerator and liquefier, Adv. Cryog. Eng. 11, 11 (1966).
[3] C. Rillo and L. Tocado, Helium-Recovery Plant, US Patent No. 8,973,397 (2015).

[4] C. Wang, Small scale helium liquefaction systems, J. Phys. Conf. Ser. 150, 012053 (2009).

[5] Model ATL160, manufactured by Quantum Design, Inc., San Diego, CA, USA.

[6] C. Chialvo, T. Sayles, J. Diederichs, S. Spagna, M. Gabal, J. Sesé, and C. Rillo, in Proceedings of the 18th International Cryocooler Conference, edited by S. D. Miller and R. G. Ross (ICC Press, Golden, CO, 2014), pp. 551-556.

[7] C. Rillo, L. Tocado, R. C. Reineman, and R. J. Warburton, Gas Liquefaction System and Method, U.S. Patent Application PCT/US2011/034842 (2011).

[8] H. O. McMahon and W. E. Gifford, A new low-temperature gas expansion cycle, Adv. Cryog. Eng. 5, 354 (1960).

[9] T. Satoh, A. Onishi, R. Li, H. Asami, and Y. Kanazawa, Development of $1.5 \mathrm{~W}$ GM cryocooler with magnetic regenerator material, Adv. Cryog. Eng. 41, 1631 (1996).

[10] E. W. Lemmon, M. L. Huber, and M. O. McLinden, NIST Standard Reference Database 23: Reference Fluid Thermodynamic and Transport Properties, version 9.1 (National Institute of Standards and Technology, Gaithersburg, 2013).

[11] T. Kuriyama, M. Takahashi, H. Nakagome, T. Eda, H. Seshake, and T. Hashimoto, Helium liquefaction by a two-stage Gifford-McMahon-cycle refrigerator using new regenerator material of $\mathrm{Er}_{3} \mathrm{Ni}$, Jpn. J. Appl. Phys. 31, L1206 (1992).

[12] R. Radebaugh, E. D. Marquardt, J. Gary, and A. O'Gallagher, in Proceedings of the 11th International Cryocooler Conference, Keystone, Colorado, 2000, edited by R. G. Ross, Jr. (Kluwer Academic, New York, 2001), p. 409.

[13] C. Wang, Intermediate cooling from pulse tube and regenerator in a $4 \mathrm{~K}$ pulse tube cryocooler, Cryogenics 48, 154 (2008).

[14] T. Prouvé, H. Godfrin, C. Gianèse, S. Triqueneaux, and A. Ravex, Experimental results on the free cooling power available on $4 \mathrm{~K}$ pulse tube coolers, J. Phys. Conf. Ser. 150, 012038 (2009).

[15] P. Schmidt-Wellenburg and O. Zimmer, Helium liquefaction with a commercial $4 \mathrm{~K}$ Gifford-McMahon cryocooler, Cryogenics 46, 799 (2006). 